\title{
Effect of double-layer structure in intramucosal gastric signet-ring cell carcinoma on lymph node metastasis: a retrospective, single-center study
}

\author{
Katsuyuki Murai ${ }^{1,2} \cdot$ Kohei Takizawa $^{2} \cdot$ Tadakazu Shimoda $^{1} \cdot$ Shougo Fujii ${ }^{1} \cdot$ Takashi Sugino $^{1} \cdot$ Masao Yoshida $^{2}$. \\ Noboru Kawata $^{2} \cdot$ Masaki Tanaka $^{2} \cdot$ Naomi Kakushima $^{2} \cdot$ Masanori Terashima $^{3} \cdot$ Hiroyuki Ono $^{2}$
}

Received: 1 July 2018 / Accepted: 25 November 2018 / Published online: 6 December 2018

(c) The International Gastric Cancer Association and The Japanese Gastric Cancer Association 2018

\begin{abstract}
Background Among all types of gastric cancer (GC), signet-ring cell carcinoma (sig-GC) accounts for 4-17\% of cases. The prognosis of early sig-GC is relatively good, with the 5-year survival rate at $99.7 \%$. However, the correlation between histological features and lymph node metastasis (LNM) among pT1a (M) sig-GC remains unclear. Sig-GC often exhibits a double-layer structure (DLS) in the intramucosal layer, demonstrating functional differentiation into the normal gastric gland. Assumedly, the loss of the differentiation makes the DLS deranged, accounting for the occurrence of submucosal invasion and LNM. This study aimed to assess the proportion of DLS, to elucidate the correlation between histological features (including DLS) and LNM status, and to determine the LNM-negative condition in pT1a (M) sig-GC.

Methods We reviewed the pathological data of 310 patients with 310 intramucosal sig-GCs who received gastrectomy with lymph node dissection. Immunohistochemistry was performed on all specimens to evaluate the presence of DLS. Furthermore, we review the clinicopathological features, including tumor size, lymphovascular invasion (LVI), ulceration (UL), and DLS results, and then statistically analyze the correlation between these features and LNM status.

Results Overall, 129 pT1a (M) sig-GCs (42\%) were DLS present. The univariate analysis revealed that "Tumor size $>20$ mm", "UL present", and "DLS absent" were significant risk factors of LNM. The multivariate logistic regression analysis revealed only "DLS absent" as statistically significant.

Conclusions "DLS absent" is a risk factor of LNM detected by the multivariate analysis. In pT1a (M), LVI absent, UL absent, tumor size $>20 \mathrm{~mm}$, sig-GC, no LNM occurred in "DLS present" cases.
\end{abstract}

Keywords Stomach neoplasms · Signet-ring cell carcinoma · Lymph node metastasis · Double-layer structure

\section{Introduction}

Gastric signet-ring cell carcinoma (sig-GC) accounts for $4-17 \%$ of all types of gastric cancer (GC) cases $[1,2]$. The prognosis of early sig-GC is good, with the 5-year survival

Tadakazu Shimoda

t.shimoda@scchr.jp

1 Division of Pathology, Shizuoka Cancer Center, 1007 Shimonagakubo, Nagaizumi, Suntogun, Shizuoka 411-8777, Japan

2 Division of Endoscopy, Shizuoka Cancer Center, Shizuoka, Japan

3 Division of Gastric Surgery, Shizuoka Cancer Center, Shizuoka, Japan rate at $99.7 \%$, whereas those of differentiated and undifferentiated adenocarcinoma are $99.1 \%$ and $97.2 \%$, respectively [3]. However, the correlation between histological features and lymph node metastasis (LNM) among pT1a (M) sig-GC remains unclear.

In the intramucosal layer, sig-GC often exhibits a doublelayer structure (DLS) [4]. While the upper layer primarily comprises intracytoplasmic abundant mucin with an eccentric nucleus, the lower layer primarily comprises poor intracytoplasmic mucin and acidophilic cytoplasm. In hematoxylin and eosin staining, the upper layer appears bright and the lower layer appears dark violet (Fig. 1). Remarkably, this structure displays functional differentiation into the normal gastric gland [4, 5]. Natsagdorj et al. reported that the presence of DLS markedly decreased with submucosal (SM) invasion [5]. In addition, we reported statistically significant 
Fig. 1 Gastric signet-ring cell carcinoma (sig-GC) often shows a double-layer structure in the intramucosal layer. The upper layer primarily comprises intracytoplasmic abundant mucin with an eccentric nucleus, and the lower layer primarily comprises poor intracytoplasmic mucin and acidophilic cytoplasm. In hematoxylin and eosin staining, the upper layer appears bright and the lower layer appears dark violet

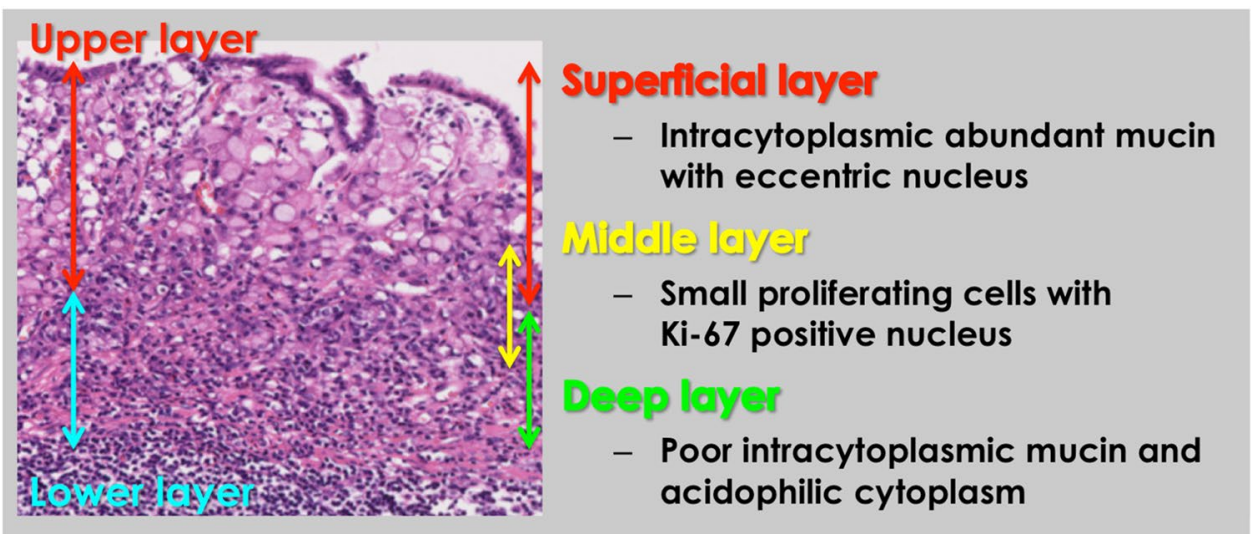

differences between the absence of DLS and occurrence of SM invasion and LNM among 534 cases of gastric carcinomas of undifferentiated type, including not only sig-GC but also poorly differentiated adenocarcinoma $(P<0.0001)$ by the univariate analysis. Among 251 DLS-present cases, 46 were SM invasion cases (18.3\%) and 18 LNM-present cases (7.2\%); conversely, among 283 DLS-absent cases, 180 were SM invasion cases (63.6\%) and 53 LNM-present cases $(18.7 \%)$ [6]. These findings suggested that the loss of the morphological and functional differentiation makes DLS deranged, accounting for the occurrence of SM invasion and LNM.

In this study, we hypothesized that among pT1a (M) sig-GC, DLS-present cases display an extremely low risk of LNM. Thus, this study aimed to assess the proportion of DLS, to elucidate the correlation between histological features (including DLS) and LNM, and to determine the LNM-negative condition among surgically resected pT1a (M) sig-GC.

\section{Methods}

\section{Patients and methods}

In this study, we reviewed the pathological data of 314 patients with $314 \mathrm{pT} 1 \mathrm{a}(\mathrm{M})$ sig-GCs who received gastrectomy with lymph node dissection (LND) at Shizuoka Cancer Center between September 2002 and December 2014. However, we excluded two patients of poor condition of specimens, and one patient of impossible cancer detection (in the new strip preparation that constituted this study's immunostaining, we could not detect the lesion, because it was minute lesion), and the other one patient of altered invasion depth (SM invasion was detected in an additional specimen). Finally, we enrolled 310 patients in this study and collected clinical data from the medical records regarding age, sex, location of the lesion, and endoscopic appearance.

\section{Pathological analysis}

We reviewed a series of pathological factors, including the tumor size, histological classification, presence of lymphatic invasion (Ly), presence of venous invasion (V), presence of ulceration (UL), immunohistochemical phenotype (e.g., gastric type, gastric and intestinal mixed type, intestinal type, and null type), distribution of cancer cells in the intramucosal layer (whole-layer type or superficial layer type), and presence of DLS. We performed D2-40 immunohistochemical staining (Nichirei, Tokyo, Japan) and Elastica van Gieson staining of all lesions to evaluate Ly and V precisely. In addition, we categorized histology into pure sig, comprising only sig, and mixed type, comprising sig, and other patterns, well-differentiated tubular adenocarcinoma (tub1), moderately differentiated tubular adenocarcinoma (tub2), poorly differentiated adenocarcinoma (por), and mucinous carcinoma (muc). Next, we categorized the distribution of cancer cells in the intramucosal layer into the following two types: wholelayer type (cancer cells extend over the entire intramucosal layer) and superficial layer type (cancer cells are confined to the superficial intramucosal layer). Moreover, we performed immunohistochemical staining MUC2 Glycoprotein (NCL-MUC2; Novocastra, UK), MUC5AC Glycoprotein (NCL-MUC5AC; Novocastra), MUC6 Glycoprotein (NCL-MUC6; Novocastra), and anti-human Ki-67 antigen (Clone MIB-I; DakoCytomation, Denmark) of all sections ( $4 \mu \mathrm{m}$ thick) from individual formalin-fixed and paraffin-embedded tumor blocks to evaluate the phenotype and presence of DLS. Furthermore, we classified the phenotype into the following four types based on the outcomes of immunostaining: gastric type (MUC2 is negative; MUC5AC and MUC6 are positive), gastric and intestinal mixed type (MUC2, MUC5AC, and MUC6 are positive), intestinal type (MUC2 is positive; MUC5AC and MUC6 are negative), and null type (MUC2, MUC5AC, and MUC6 are negative). 


\section{Definition of DLS}

In the normal mucosa, foveolar epitheliums, which are positive for MUC5AC, spread over the superficial intramucosal layer. In addition, pyloric glands and mucous neck cells, which are positive for MUC6, spread in the deep intramucosal layer. Proliferating cells, which are positive for antihuman Ki-67 antigen, spread in the borderline between foveolar epitheliums and pyloric glands, mucous neck cells (Fig. 2). DLS that is often observed in the intramucosal layer of sig-GC displays functional differentiation into the normal gastric gland. As shown in Fig. 3, from the top to the bottom, each immunohistochemical staining positive area is arranged in the order of the red line (MUC5AC-positive area), green line (MUC6-positive area), and yellow line (Ki-67-positive area) and localized to the borderline similar to the normal mucosa; such cases are classified as "DLS-present cases". However, MUC5AC and MUC6 do not always exhibit positive reactions in sig-GC. Thus, in this study, DLS-present cases are defined as cases that fulfill the following conditions: (a) MUC5AC exhibits a positive reaction only in the superficial intramucosal layer; (b) MUC6 exhibits a positive reaction only in the deep intramucosal layer; and (c) Ki-67 exhibits a positive reaction limited to the middle layer. In Fig. 4, the red line (MUC5AC-positive area) overlaps the green line (MUC6-positive area), and the yellow line (Ki67-positive area) spreads widely; such cases that do not fulfill the conditions mentioned above are classified as "DLSabsent cases". When we assessed the presence or absence of DLS, we used each section that had the tumor maximum infiltration units, because derangement of DLS was assumed to occur first in the area. In addition, the presence or the absence of DLS was determined by superiority in the same way as diagnosing predominant pathological type in dairy everyday clinical practice. If the DLS-present area spread more widely than the DLS-absent area, the case was diagnosed as DLS present. Furthermore, the sections were examined by two pathologists (K.M. and T.S.), and any conflict was resolved by mutual consensus.

\section{Measurements}

In this study, we assessed the proportion of DLS among pT1a (M) sig-GC, the relevance of the histological features and LNM, and the LNM-negative condition. This study protocol was approved by the Institutional Review Board of Shizuoka Cancer Center (approval number: 27-J111). Informed consent from enrolled patients was waived by the requirement of the approving authority.

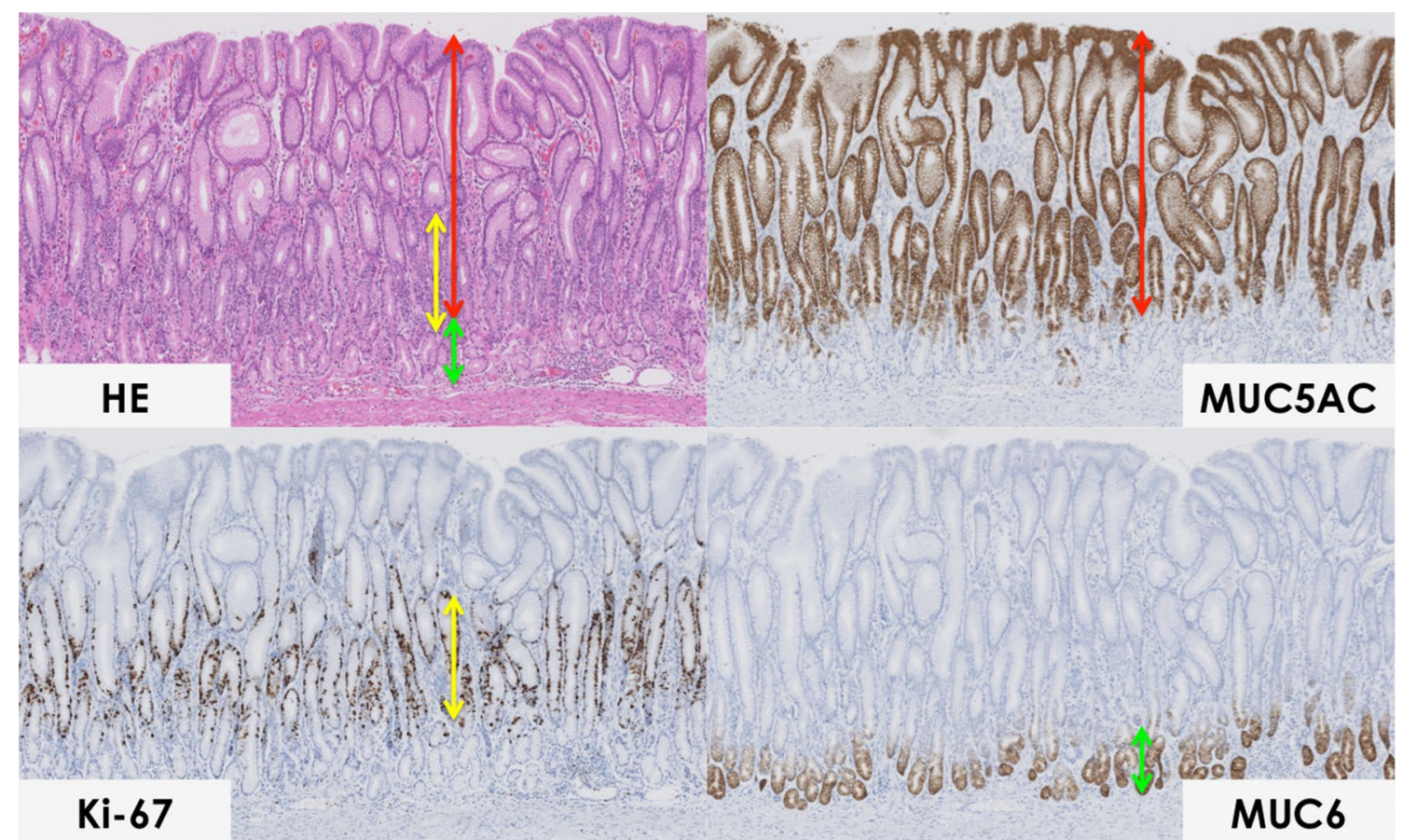

Fig. 2 In the normal mucosa, foveolar epitheliums, which are positive for MUC5AC (red line), spread the superficial intramucosal layer. In addition, pyloric glands and mucous neck cells, which are positive for MUC6 (green line), spread in the deep intramucosal layer. Proliferating cells, which are positive for anti-human Ki-67 antigen (yel- low line), spread in the borderline between foveolar epitheliums and pyloric glands, mucous neck cells. From the top to the bottom, each immunohistochemical staining positive area is arranged in the order of the red and green lines. The yellow line is localized to the borderline 


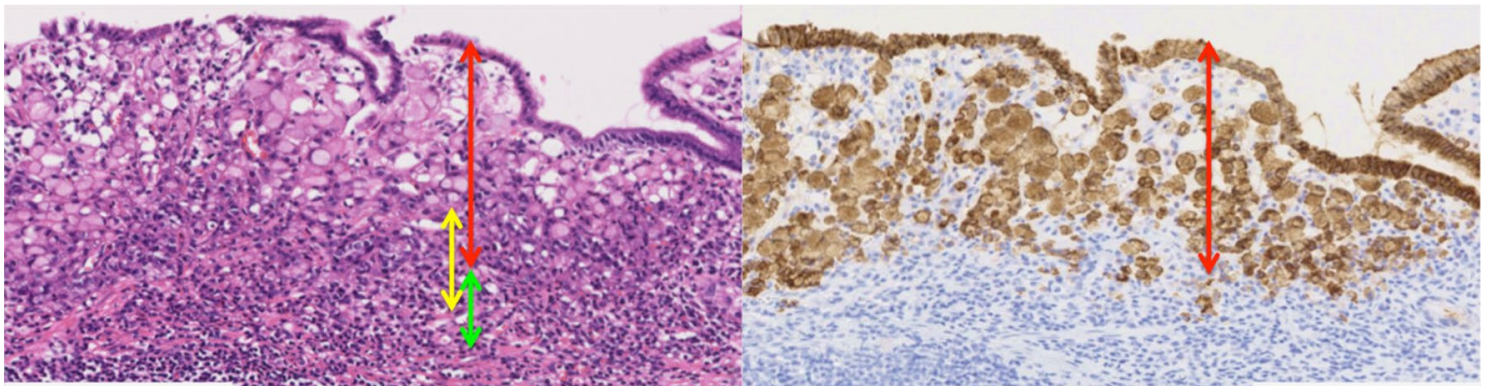

HE

MUC5AC

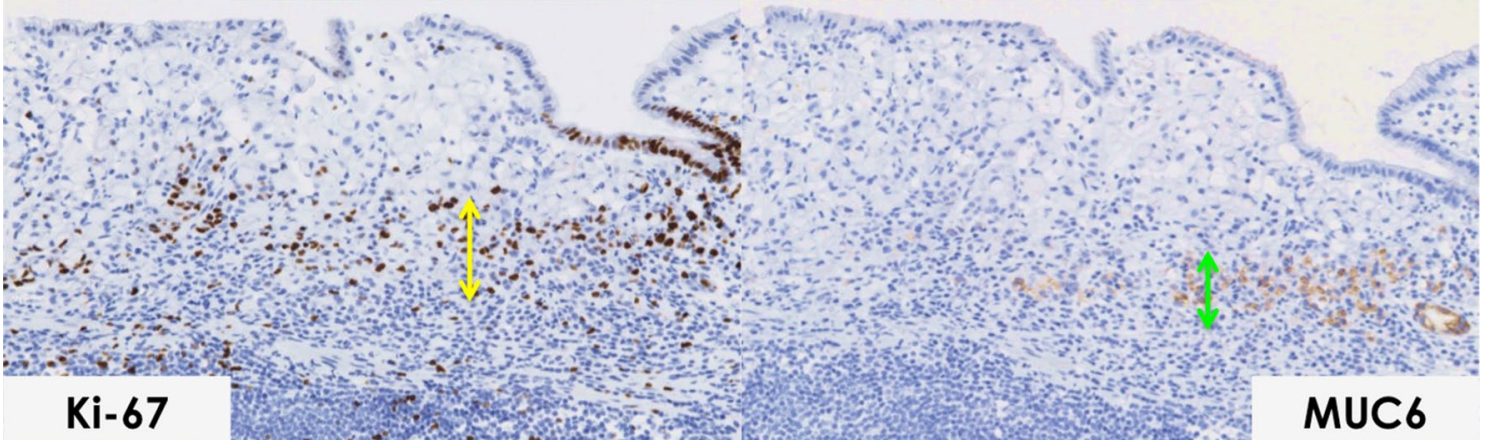

Fig. 3 In this case, from the top to the bottom, they are arranged in the order of the red and green lines, and the yellow line is localized to the borderline, similar to the normal mucosa (Fig. 2). Such cases are classified as "double-layer structure present cases"

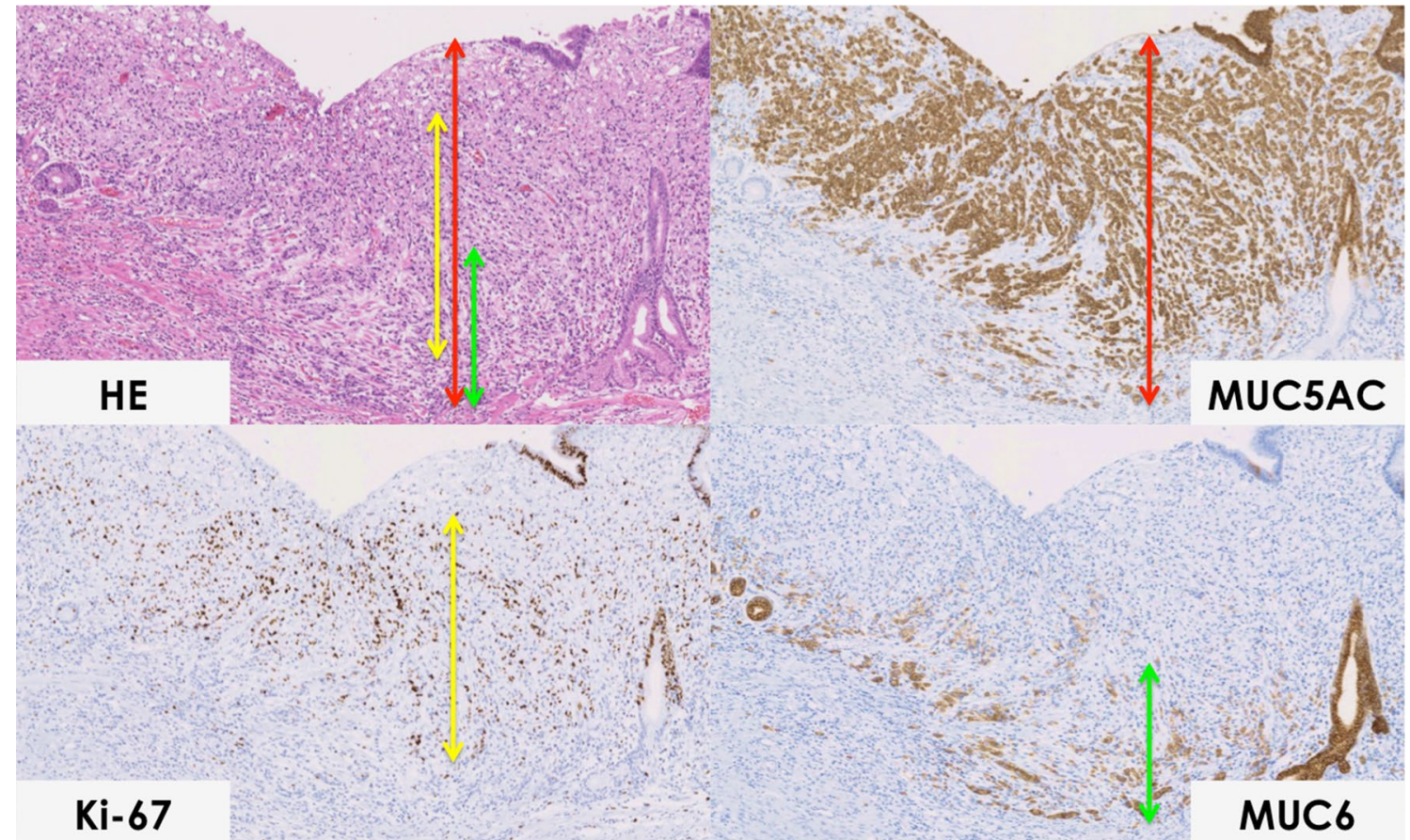

Fig. 4 In this case, the red line overlaps the green line, and the yellow line spreads broadly. Such cases are classified as "double-layer structure absent cases" 


\section{Statistical analysis}

The clinical and pathological data were analyzed using EZR version 1.36 (Saitama Medical Center, Jichi Medical University, Saitama, Japan) [7], which is a graphical user interface for R (The R Foundation for Statistical Computing, Vienna, Austria). Precisely, it is a modified version of $\mathrm{R}$ commander designed to add statistical functions frequently used in biostatistics. In addition, we used Fisher's exact test and logistic regression analysis for the statistical analysis of the correlation between clinicopathological variables and the LNM status. Statistical significance was defined as $P<0.10$ in the univariate analysis and $P<0.05$ in the multivariate logistic regression analysis.

\section{Results}

In this study, we included 310 lesions (310 patients) of pT1a (M) sig-GCs. Table 1 summarizes the clinicopathological features of these 310 lesions. The median age of patients was 61 years, and half of the patients were male. Most lesions were located in the middle (59\%) or lower portion of stomach (32\%). In addition, the histological type was pure sig $(60 \%)$ and mixed type (40\%). Moreover, Ly was present in 5 lesions (2\%). In addition, UL was present in 131 lesions (42\%); however, $\mathrm{V}$ was absent in all lesions. The distribution of cancer cells among the intramucosal layers was whole-layer type in 200 lesions (65\%). LNM was present in 21 lesions (7\%). Furthermore, immunostaining revealed that most lesions were classified into the gastric type (51\%) or gastric and intestinal mixed type (45\%). Among our cases, 129 lesions (42\%) were DLS present.

We performed the univariate analysis and multivariate logistic regression analysis to assess the LNM risk (Table 2). The univariate analysis revealed that "Tumor size $>20 \mathrm{~mm}$ ", "UL present", and "DLS absent" were significant risk factors for LNM $(P=0.02,0.07$, and 0.01 , respectively). The multivariate logistic regression analysis of the risk factor for LNM revealed only "DLS absent" as statistically significant [odds ratio [OR], 4.93; 95\% confidence interval (CI) 1.41-17.3; $P=0.01]$. In addition, "Tumor size $>20$ mm" did not exhibit a statistical significance (OR 6.99; 95\% CI $0.90-54.1 ; P=0.06$ ), similar to "UL present" (OR 1.92 ; 95\% CI $0.75-4.91, P=0.17)$. Considering the LNMnegative condition, in pT1a (M), UL absent, size $\leq 20 \mathrm{~mm}$, sig-GC, which fulfilled the criteria defined by the Japanese guidelines [8], no LNM was reported. Furthermore, in pT1a (M), lymphovascular invasion (LVI) absent, UL absent, size $>20 \mathrm{~mm}$, and sig-GC, no LNM cases with "DLS present" were reported (Fig. 5).
Table 1 Patient and lesion characteristics

\begin{tabular}{|c|c|}
\hline & $\begin{array}{l}310 \text { patients, } \\
310 \text { lesions }\end{array}$ \\
\hline Median age, years (range) & $61(19-87)$ \\
\hline \multicolumn{2}{|l|}{ Gender $[n(\%)]$} \\
\hline Male & $151(49)$ \\
\hline Female & $159(51)$ \\
\hline \multicolumn{2}{|l|}{ Location $[n(\%)]$} \\
\hline Upper portion of stomach & $27(9)$ \\
\hline Middle portion of stomach & $184(59)$ \\
\hline Lower portion of stomach & $99(32)$ \\
\hline Median tumor size, mm (range) & $32(1-182)$ \\
\hline \multicolumn{2}{|l|}{ Histology $[n(\%)]$} \\
\hline Pure sig & $187(60)$ \\
\hline Mixed type & $123(40)$ \\
\hline \multicolumn{2}{|l|}{ Lymphatic invasion $[n(\%)]$} \\
\hline Present & $5(2)$ \\
\hline Absent & $305(98)$ \\
\hline \multicolumn{2}{|l|}{ Venous invasion $[n(\%)]$} \\
\hline Present & $0(0)$ \\
\hline Absent & $310(100)$ \\
\hline \multicolumn{2}{|l|}{ Ulceration $[n(\%)]$} \\
\hline Present & $131(42)$ \\
\hline Absent & $179(58)$ \\
\hline \multicolumn{2}{|l|}{ Lymph node metastasis [ $n(\%)]$} \\
\hline Present & $21(7)$ \\
\hline Absent & $289(93)$ \\
\hline \multicolumn{2}{|l|}{ Phenotype $[n(\%)]$} \\
\hline Gastric type & $157(51)$ \\
\hline Mixed type & $138(45)$ \\
\hline Intestinal type & $8(3)$ \\
\hline Null type & $7(2)$ \\
\hline \multicolumn{2}{|l|}{ Distribution $[n(\%)]$} \\
\hline Whole layer & $200(65)$ \\
\hline Superficial layer & $110(35)$ \\
\hline \multicolumn{2}{|l|}{ Double-layer structure $[n(\%)]$} \\
\hline Present & $129(42)$ \\
\hline Absent & $181(58)$ \\
\hline
\end{tabular}

Mixed type gastric and intestinal mixed type, Distribution the distribution of cancer cells in the intramucosal layer

\section{Discussion}

Recent advancements in endoscopic technologies, including early diagnosis and endoscopic treatment, have increased the number of patients with early gastric cancer (EGC) treated with endoscopic submucosal dissection (ESD) throughout the world. Based on the pathological results of ESD, it is considered as a curative resection when the lesion is removed en-bloc with negative margins, LVI is absent, and the criteria defined per the Japanese guidelines [8] are 
Table 2 Univariate analysis and multivariate logistic regression analysis for the risk of LNM

\begin{tabular}{|c|c|c|c|c|}
\hline & LNM positive $(n=21)$ & LNM negative $(n=289)$ & OR $(95 \% \mathrm{CI})$ & $P$ \\
\hline \multicolumn{5}{|c|}{ Univariate analysis for the risk of LNM } \\
\hline \multicolumn{5}{|l|}{ Age $[n(\%)]$} \\
\hline$<60$ years & $12(57)$ & $128(44)$ & Reference & \multirow[t]{2}{*}{0.27} \\
\hline$\geq 60$ years & $9(43)$ & $161(56)$ & $0.60(0.24-1.46)$ & \\
\hline \multicolumn{5}{|c|}{ Gender $[n(\%)]$} \\
\hline Male & $9(43)$ & $142(49)$ & Reference & \multirow[t]{2}{*}{0.66} \\
\hline Female & $12(57)$ & $147(51)$ & $1.29(0.53-3.15)$ & \\
\hline \multicolumn{5}{|c|}{ Location $[n(\%)]$} \\
\hline M, L & $18(86)$ & $265(92)$ & Reference & \multirow[t]{2}{*}{0.41} \\
\hline $\mathrm{U}$ & $3(14)$ & $24(8)$ & $1.84(0.51-6.70)$ & \\
\hline \multicolumn{5}{|l|}{ Size $[n(\%)]$} \\
\hline$\leq 20 \mathrm{~mm}$ & $1(5)$ & $79(27)$ & Reference & \multirow[t]{2}{*}{$0.02 *$} \\
\hline$>20 \mathrm{~mm}$ & $20(95)$ & $210(73)$ & $7.52(0.99-57.00)$ & \\
\hline \multicolumn{5}{|c|}{ Histology $[n(\%)]$} \\
\hline Pure sig & $12(57)$ & $175(61)$ & Reference & \multirow[t]{2}{*}{0.82} \\
\hline Mixed type & $9(43)$ & $114(39)$ & $1.15(0.47-2.82)$ & \\
\hline \multicolumn{5}{|l|}{ Ly $[n(\%)]$} \\
\hline Absent & $20(95)$ & $285(99)$ & Reference & \multirow[t]{2}{*}{0.30} \\
\hline Present & $1(5)$ & $4(1)$ & $3.56(0.38-33.40)$ & \\
\hline \multicolumn{5}{|l|}{$\mathrm{UL}[n(\%)]$} \\
\hline Absent & $8(14)$ & $171(59)$ & Reference & \multirow[t]{2}{*}{$0.07 *$} \\
\hline Present & $13(86)$ & $118(41)$ & $2.35(0.95-5.86)$ & \\
\hline \multicolumn{5}{|c|}{ Distribution $[n(\%)]$} \\
\hline Superficial & $4(19)$ & $106(37)$ & Reference & \multirow[t]{2}{*}{0.16} \\
\hline Whole & $17(81)$ & $183(63)$ & $2.46(0.81-7.51)$ & \\
\hline \multicolumn{5}{|l|}{$\operatorname{DLS}[n(\%)]$} \\
\hline Present & $3(14)$ & $126(44)$ & Reference & \multirow[t]{2}{*}{$0.01 *$} \\
\hline \multirow[t]{2}{*}{ Absent } & $18(86)$ & $163(56)$ & $4.64(1.34-16.10)$ & \\
\hline & & OR & $95 \% \mathrm{CI}$ & $P$ \\
\hline \multicolumn{5}{|c|}{ Multivariate logistic regression analysis for risk of LNM } \\
\hline Size & $>20 \mathrm{~mm}$ & 6.99 & $0.90-54.10$ & 0.06 \\
\hline UL & Present & 1.92 & $0.75-4.91$ & 0.17 \\
\hline DLS & Absent & 4.93 & $1.41-17.30$ & $0.01 *$ \\
\hline
\end{tabular}

Distribution the distribution of cancer cells in the intramucosal layer, $D L S$ double-layer structure, $L$ lower stomach, $L y$ lymphatic invasion, $L N M$ lymph node metastasis, $M$ middle stomach, $O R$ odds ratio, Superficial superficial layer, $U$ upper stomach, $U L$ ulceration, Whole whole layer

*Statistically significant

fulfilled, because the LNM risk for such lesions is extremely low $[9,10]$. However, if a lesion does not fulfill these conditions, the resection is considered as a noncurative ESD, and additional gastrectomy with LND is considered as the standard treatment. In contrast, the LNM frequency among additional gastrectomy in patients with a noncurative ESD is low. Previously, we reported that LNM was found in $9.3 \%$ (30/323) of patients who underwent additional gastrectomy after a noncurative ESD [11]. In contrast, additional gastrectomy could be an overtreatment for nearly $90 \%$ of patients with a noncurative ESD. Hence, it is imperative to determine the factors that contribute to LNM to avoid unnecessary gastrectomy.

This study focused on pT1a (M) sig-GC. Previously, several studies have reported that lower rates of LNM and favorable prognoses correlated with early sig-GC compared with other undifferentiated EGCs [12-14]. In addition, a recent study reported that the LNM rate in pT1a (M) sig-GC was as low as that in well-differentiated EGCs [15]. Furthermore, the incidence of LNM in pT1a (M) sig-GC has been reported to be between $1.6 \%$ and $6.3 \%$ [3, 15-17]. In this study, however, the LNM rate was $6.8 \%$ (21/310). 
Fig. 5 Considering the lymph node metastasis (LNM)-negative condition, in pT1a (M), lymphovascular invasion (LVI) absent, ulceration (UL) absent, size $\leq 20 \mathrm{~mm}$, sig-GC, which fulfilled the criteria defined by the Japanese guidelines, there was no LNM case was reported. In addition, in pT1a (M), LVI absent, UL absent, tumor size $>20 \mathrm{~mm}$, sig-GC, no LNM cases occurred with "Doublelayer structure present"

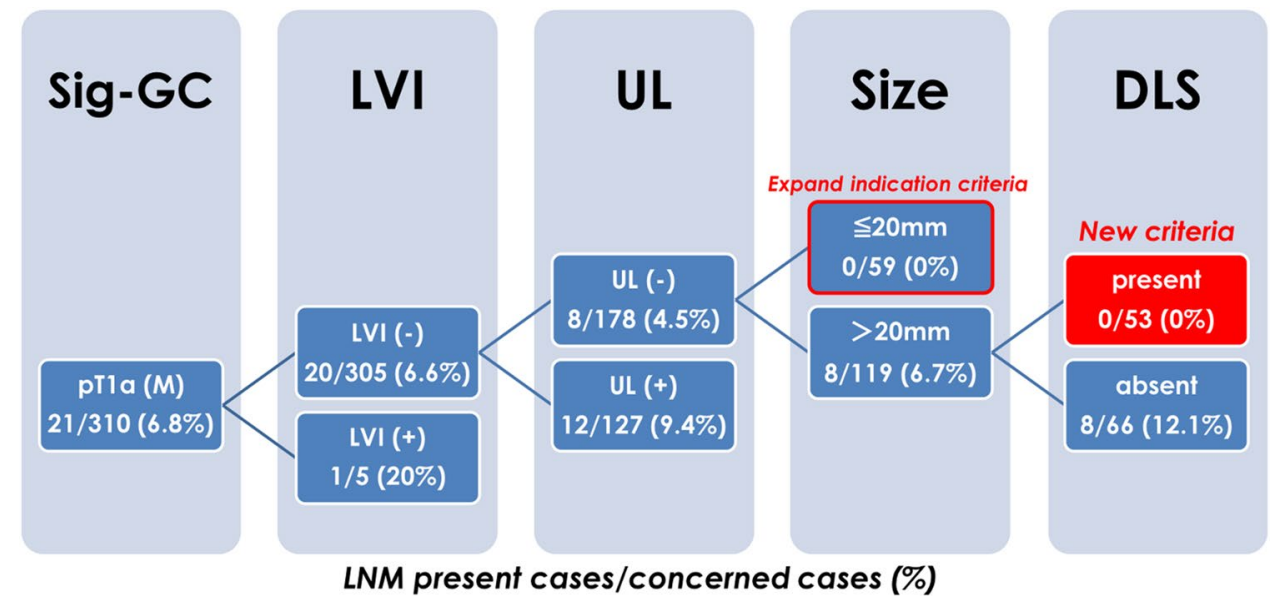

LVI: Lymphovascular invasion, UL: Ulceration, DLS: Double-layer structure, LNM: Lymph node metastasis
In addition, we focused on DLS as a new risk factor of LNM. In this study, the proportion of DLS among intramucosal EGCs was 42\% (129/310), and "DLS absent" exhibited a statistical significance with not only the univariate analysis, similar to a previous report [6], but also the multivariate logistic regression analysis risk factor of LNM. The correlation between DLS and LNM seemed more probable. Moreover, in pT1a (M), LVI absent, UL absent, tumor size $>20 \mathrm{~mm}$, and sig-GC, no LNM case with "DLS present" was reported (Fig. 5). Based on the pathological results of ESD, UL-absent cT1a undifferentiated-type carcinomas with $<2 \mathrm{~cm}$ in diameter are considered as a curative resection when a lesion is removed en-bloc with negative margins and LVI is absent. This study highlighted the possibility that even if the pathological results of ESD do not fulfill these conditions, DLS-present cases do not need an additional gastrectomy, because the LNM risk for such lesions is assumedly extremely low. Recently, Pyo et al. reported the risk-scoring system to estimate LNM referring the largest number (1544) of patients with pT1a (M) sigGCs [18]. However, they did not evaluate UL. Reported, UL exerts a great impact on LNM [9]. Indeed, in this study, the univariate analysis revealed that "Tumor size $>20 \mathrm{~mm}$ " and "UL present" are significant risk factors of LNM. Although the multivariate logistic regression analysis risk factor of LNM did not exhibit a statistical significance in this study, UL should be assessed when investigating risk factors of LNM among EGCs.

Among patients categorized as having noncurative gastric ESD, the multivariate logistic regression analysis revealed LVI as an independent risk factor for LNM (OR 8.57; 95\% CI 2.76-38.14; $P<0.0001$ ) [9]. Another study reported similar findings regarding LVI (OR 6.7; 95\% CI 5.0-8.9; $P<0.001)$ [19]. In this study, although positive ratio of LNM among LVI-positive cases was 20\% (1/5 cases), LVI did not exhibit a statistical significance, which could be attributed to the limited number of LVI-positive cases (5 cases) included. Notably, we enrolled only 5 LVIpositive cases, because subjects in this study were only pT1a sig-GCs, which did not include SM invasive GCs. Considering the high odds of LNM for LVI, patients who received a noncurative ESD without LVI should be tested for the presence of DLS to avoid unnecessary additional surgery.

This study has several limitations. First, this study was a retrospective, single-center analysis. Thus, a large, multicenter, prospective study is warranted. Second, several cases of conflict occurred between pathologists in assessing the presence of DLS. Notably, it was challenging to evaluate the presence of DLS, especially in UL-positive cases, because most infiltration units in UL-positive cases existed adjacent to an ulcer, and the number of tumor cells was low in the area. Hence, the diagnosis algorithm design (Fig. 5) may be acceptable. In other words, based on the criteria defined by the Japanese guidelines [8], first, we evaluate LVI, UL, and tumor size, and then only in LVI absent, UL absent, tumor size $>20 \mathrm{~mm}$, sig-GC cases, we add immunohistochemical staining (MUC2, MUC5AC, and MUC6) to evaluate the presence of DLS.

In conclusion, the proportion of DLS among intramucosal EGCs was $42 \%$ in this study. The univariate analysis revealed that "Tumor size $>20 \mathrm{~mm}$ ", "UL present", and "DLS absent" are significant risk factors of LNM among pT1a (M) sig-GC. However, the multivariate logistic regression analysis of the risk factor of LNM revealed only "DLS absent" as statistically significant. In pT1a (M), LVI absent, UL absent, tumor size $>20 \mathrm{~mm}$, and sig-GC, no LNM cases were "DLS present" cases. Thus, this study suggests that additional gastrectomy with LND may not be required for such cases. Hence, we strongly recommend an assessment about DLS with immunostaining in pT1a (M), LVI absent, UL absent, size $>20 \mathrm{~mm}$, and sig-GC cases. 


\section{Compliance with ethical standards}

Conflict of interest The authors have no conflict of interest to declare.

Ethical standards All procedures were followed in accordance with the ethical standards of the Institutional Review Board of Shizuoka Cancer Center (approval number: 27-J111) and with the Helsinki Declaration of 1964 and later versions. Informed consent from enrolled patients was waived by the requirement of the approving authority.

\section{References}

1. Maehara Y, Sakaguchi Y, Moriguchi S, Orita H, Korenaga D, Kohnoe S, et al. Signet ring cell carcinoma of the stomach. Cancer. 1992;69:1645-50.

2. Otsuji E, Yamaguchi T, Sawai K, Takahashi T. Characterization of signet ring cell carcinoma of the stomach. J Surg Oncol. 1998;67:216-20.

3. Ha TK, An JY, Youn HK, Noh JH, Sohn TS, Kim S. Indication for endoscopic mucosal resection in early signet ring cell gastric cancer. Ann Surg Oncol. 2008;15:508-13.

4. Sugihara H, Hattori T, Fukuda M, Fujita S. Cell proliferation and differentiation in intramucosal and advanced signet ring cell carcinomas of the human stomach. Virchows Arch A Pathol Anat Histopathol. 1987;411:117-27.

5. Natsagdorj L, Sugihara H, Bamba M, Hattori T. Intratumoural heterogeneity of intestinal expression reflects environmental induction and progression-related loss of induction in undifferentiatedtype gastric carcinomas. Histopathology. 2008;53:685-97.

6. Takizawa K, Shimoda T, Nakanishi Y, Taniguchi H, Oda I, Gotoda T. Expanded indication for endoscopic resection from the pathological viewpoint - the possibility of sm invasion by undifferentiated type early gastric cancer. In: The Research Society for Early Gastric Cancer (ed). Stomach and Intestine. Tokyo: Igaku-Shoin Ltd Publisher; 2006. pp. 9-17.

7. Kanda Y. Investigation of the freely-available easy-to-use software "EZR" (Easy R) for medical statistics. Bone Marrow Transplant. 2013:48,452-458.

8. Ono H, Yao K, Fujishiro M, Oda I, Nimura S, Yahagi N, et al. Guidelines for endoscopic submucosal dissection and endoscopic mucosal resection for early gastric cancer. Dig Endosc. 2016;28:3-15.

9. Gotoda T, Yanagisawa A, Sasako M, Ono H, Nakanishi Y, Shimoda $\mathrm{T}$, et al. Incidence of lymph node metastasis from early gastric cancer: estimation with a large number of cases at two large centers. Gastric Cancer. 2000;3:219-25.

10. Hirasawa T, Gotoda T, Miyata S, Kato Y, Shimoda T, Taniguchi $\mathrm{H}$, et al. Incidence of lymph node metastasis and the feasibility of endoscopic resection for undifferentiated-type early gastric cancer. Gastric Cancer. 2009;12:148-52.

11. Kawata N, Kakushima N, Takizawa K, Tanaka M, Makuuchi R, Tokunaga M, et al. Risk factors for lymph node metastasis and long-term outcomes of patients with early gastric cancer after non-curative endoscopic submucosal dissection. Surg Endosc. 2017;31:1607-16.

12. Hyung WJ, Noh SH, Lee JH, Huh JJ, Lah KH, Chol SH, et al. Early gastric carcinoma with signet ring cell histology. Cancer. 2002;94:78-83.

13. Chiu CT, Kuo CJ, Yeh TS, Hsu JT, Liu KH, Yeh CN, et al. Early signet ring cell gastric cancer. Dig Dis Sci. 2011;56:1749-56.

14. Kunisaki C, Shimada H, Nomura M, Matsuda G, Otsuka Y, Akiyama $\mathrm{H}$. Therapeutic strategy for signet ring cell carcinoma of the stomach. Br J Surg. 2004;91:1319-24.

15. Lee SH, Jee SR, Kim JH, Seol SY. Intramucosal gastric cancer: the rate of lymph node metastasis in signet ring cell carcinoma is as low as that in well- differentiated adenocarcinoma. Eur J Gastroenterol Hepatol. 2015;27:170-4.

16. Kim HM, Pak KH, Chung MJ, Cho JH, Hyung WJ, Noh SH, et al. Early gastric cancer of signet ring cell carcinoma is more amenable to endoscopic treatment than is early gastric cancer of poorly differentiated tubular adenocarcinoma in select tumor conditions. Surg Endosc. 2011;25:3087-93.

17. Kim BS, Oh ST, Yook JH, Kim BS. Signet ring cell type and other histologic types: differing clinical course and prognosis in T1 gastric cancer. Surgery. 2014;155:1030-5.

18. Pyo JH, Shin CM, Lee H, Min BH, Lee JH, Kim SM, et al. A risk-prediction model based on lymph-node metastasis for incorporation into a treatment algorithm for signet ring cell-type intramucosal gastric cancer. Ann Surg. 2016;264:1038-43.

19. Sekiguchi M, Oda I, Taniguchi H, Suzuki H, Morita S, Fukagawa $\mathrm{T}$, et al. Risk stratification and predictive risk-scoring model for lymph node metastasis in early gastric cancer. J Gastroenterol. 2016;51:961-70. 